\title{
THE DENSITY OF ZEROS OF FORMS FOR WHICH WEAK APPROXIMATION FAILS
}

\author{
D. R. HEATH-BROWN
}

\begin{abstract}
The weak approximation principle fails for the forms $x^{3}+y^{3}+z^{3}=$ $k w^{3}$, when $k=2$ or 3 . The question therefore arises as to what asymptotic density one should predict for the rational zeros of these forms. Evidence, both numerical and theoretical, is presented, which suggests that, for forms of the above type, the product of the local densities still gives the correct global density.
\end{abstract}

Let $f\left(x_{1}, \ldots, x_{n}\right) \in \mathbb{Q}\left[x_{1}, \ldots, x_{n}\right]$ be a rational form. We say that $f$ satisfies the weak approximation principle if the following condition holds.

(WA) Given an $\varepsilon>0$, a finite set $S$ of places of $\mathbb{Q}$, and zeros

$$
\left(x_{1}^{\nu}, \ldots, x_{n}^{\nu}\right) \in \mathbb{Q}_{\nu}^{n}
$$

of the form $f$, we can find a rational zero $\left(x_{1}, \ldots, x_{n}\right)$ of $f$ such that

$$
\left|x_{i}-x_{i}^{\nu}\right|_{\nu}<\varepsilon \text { for } 1 \leq i \leq n \text { and } \nu \in S .
$$

Alternatively, we may write $X(K)$ for the points on the hypersurface $f=0$ whose coordinates lie in the field $K$, and consider the product

$$
\Pi_{S}=\prod_{\nu \in S} X\left(\mathbb{Q}_{\nu}\right),
$$

with the product topology. Then (WA) says that for any finite set $S$ of places, the image of $X(\mathbb{Q})$ in $\Pi_{S}$, under the diagonal embedding, is dense. Thus, for example, nonsingular quadratic forms which represent zero satisfy (WA) when $n \geq 3$. However, the cubic forms $x^{3}+y^{3}+z^{3}-k w^{3} \quad(k=2,3)$ do not satisfy (WA), as we shall show.

Conjectures about the density of zeros of $f$ are often made by calculating the product of all the local densities. These conjectures can sometimes be proved using the Hardy-Littlewood circle method, and the product of densities one obtains is just the singular series multiplied by the singular integral. However, such conjectures must seem highly dubious when (WA) fails. For then one knows that there are subsets of $\Pi_{S}$ where there are a positive density of points, but no rational points. Two possible reformulations of the conjecture on the global density seem plausible. Either one may make no adjustment, in the expectation that the density of rational points in the admissible part of $\Pi_{S}$ is

Received by the editor March 5, 1991.

1991 Mathematics Subject Classification. Primary 11D25; Secondary 11G35, 11 P55.

Key words and phrases. Cubic surfaces, weak approximation, Brauer-Manin obstruction, HardyLittlewood formula, asymptotic estimates. 
correspondingly increased; or one may reduce the product of local densities by removing those parts of $\Pi_{S}$ which the failure of (WA) excludes. When the Hasse Principle fails for $f$, we must clearly follow the second procedure. This would be the case for the example $5 x^{3}+12 y^{3}+9 z^{3}+10 w^{3}$ of Cassels and Guy [2], for instance.

The purpose of this paper is to investigate further the above options, principally through an examination of the forms $x^{3}+y^{3}+z^{3}-k w^{3}$. We first prove:

Theorem 1. If $(x, y, z, w) \in \mathbb{Z}^{4}$ is a primitive solution of $x^{3}+y^{3}+z^{3}=$ $2 w^{3}$ (so that $\left.\operatorname{hcf}(x, y, z, w)=1\right)$, then one of $x, y, z$ is divisible by 6 . If $(x, y, z, w) \in \mathbb{Z}^{4}$ is a primitive solution of $x^{3}+y^{3}+z^{3}=3 w^{3}$, then either $x \equiv y \equiv z(\bmod 9)$ or one of $x, y, z$ is divisible by 9 .

This demonstrates the failure of (WA) for the above forms. The first form can have no rational zero close to both $(0,1,1,1) \in \mathbb{Q}_{2}^{4}$ and $(1,0,1,1) \in \mathbb{Q}_{3}^{4}$. Similarly, the second form has no rational zero close to $(-2,1,4, \sqrt[3]{19}) \in \mathbb{Q}_{3}^{4}$.

In a letter to the author, Professor Colliot-Thélène has shown that the above congruence restrictions are exactly those implied by the Brauer-Manin obstruction. Moreover, for the general equation $x^{3}+y^{3}+z^{3}=k w^{3}$, with a noncube integer $k$, there is always a nontrivial obstruction, eliminating two-thirds of the adèlic points.

The proof of the theorem is based on a method of Cassels [1], and uses cubic reciprocity in $\mathbb{Q}(\omega)$, where $\omega=(-1+\sqrt{-3}) / 2$. Let $k=2$ or 3 as appropriate, and suppose that $x+y \omega$ has a prime factor $\pi$ in $\mathbb{Q}(\omega)$. We write $p$ for the rational prime above $\pi$, and we suppose that $p \nmid 3 k$. If $p$ is inert, then

$$
(k / \pi)_{3}=1
$$

automatically, and otherwise the congruence $z^{3} \equiv k w^{3}(\bmod \pi)$ implies (1) unless $p \mid \operatorname{hcf}(z, w)$. In the latter case, suppose that $\pi^{e} \| x+y \omega$. Since $x, y$, $z$, and $w$ are coprime, we have $p \nmid \operatorname{hcf}(x, y)$, so that $\pi \nmid x+y, x+y \omega^{2}$. Thus, $\pi^{e} \| x^{3}+y^{3}$, whence $\pi^{e} \| z^{3}-k w^{3}$. Hence, either (1) holds or $3 \mid e$. We may therefore conclude that

$$
\left(k / \pi^{e}\right)_{3}=1
$$

whenever $\pi^{e} \| x+y \omega$ and $\pi \nmid 3 k$.

If $k=2$, then exactly one of $x, y, z$ will be even, so that $2 \nmid x+y \omega$. Moreover, a congruence $(\bmod 9)$ shows that 3 divides exactly one of $x, y$, $z$ (y say). Then $\operatorname{hcf}(x+y \omega, 3)=1$ and (2) holds for all prime factors of $x+y \omega$. It follows that

$$
\left(\frac{2}{x+y \omega}\right)_{3}=1
$$

on multiplying up the various relations (2). The law of cubic reciprocity now yields

$$
\left(\frac{x+y \omega}{2}\right)_{3}=1,
$$

since $3 \mid y$. This implies that $y$ must be even, and the theorem, for this case, follows. 
If $k=3$, then a congruence $(\bmod 3)$ shows that either

$$
x \equiv y \equiv z \equiv \pm 1 \quad(\bmod 3),
$$

or 3 divides exactly one of $x, y, z$ ( $y$ say). In the former case, $x+y \omega$ has a primary associate $x \omega+y \omega^{2}$, and the fact that

$$
\left(\frac{3}{x \omega+y \omega^{2}}\right)_{3}=1
$$

yields

$$
x \omega+y \omega^{2} \equiv \pm 1, \pm 2, \text { or } \pm 4(\bmod 9),
$$

by the supplement to the law of reciprocity. Hence $x \equiv y(\bmod 9)$, and, by symmetry, $x \equiv y \equiv z(\bmod 9)$. In case $3 \mid y$ and $3 \nmid x z$, then $x+y \omega$ is primary, and an analogous argument yields $9 \mid y$. This proves the theorem in the second case.

For a fixed $k \neq 0$, the local density of zeros at a finite place $p$ is given by

$$
\sigma_{p, k}=\lim _{n \rightarrow \infty} p^{-3 n} N\left(p^{n}\right),
$$

where

$$
\begin{array}{r}
N\left(p^{n}\right)=N_{k}\left(p^{n}\right)=\#\left\{(x, y, z, w)\left(\bmod p^{n}\right): p \nmid \operatorname{hcf}(x, y, z, w),\right. \\
\left.x^{3}+y^{3}+z^{3} \equiv k w^{3}\left(\bmod p^{n}\right)\right\} .
\end{array}
$$

This expression can be deduced, for example, by considering the major arc contribution to a suitable integral. Thus, if we write $e(\alpha)=\exp (2 \pi i \alpha)$, then

$$
\begin{array}{r}
R_{k}(N)=\#\left\{(x, y, z, w) \in \mathbb{Z}^{4}:|x|,|y|,|z| \leq N, \operatorname{hcf}(x, y, z, w)=1,\right. \\
\left.x^{3}+y^{3}+z^{3}=k w^{3}\right\}
\end{array}
$$

is given by

$$
\begin{aligned}
\int_{0}^{1} & \sum_{\substack{|x|,|y|,|z| \leq N \\
|w| \leq 2 N}}^{(1)} e\left(\alpha\left(x^{3}+y^{3}+z^{3}-k w^{3}\right)\right) d \alpha \\
= & \sum_{d=1}^{\infty} \mu(d) \int_{0}^{1} \sum_{\substack{|x|,|y|,|z| \leq N \\
|w| \leq 2 N}}^{(2)} e\left(\alpha\left(x^{3}+y^{3}+z^{3}-k w^{3}\right)\right) d \alpha,
\end{aligned}
$$

where $\sum^{(1)}$ denotes the condition $\operatorname{hcf}(x, y, z, w)=1$, and $\sum^{(2)}$ denotes the conditions $d \mid x, y, z, w$ and $(x, y, z, w) \neq(0,0,0,0)$. For the abovementioned region, the real density of solutions will be $\sigma_{\infty, k}=k^{-1 / 3} / I$, where

$$
I=\frac{1}{3} \int_{-1}^{1} \int_{-1}^{1} \int_{-1}^{1} \frac{d x d y d z}{\left(x^{3}+y^{3}+z^{3}\right)^{2 / 3}}
$$

We shall not reproduce the calculation of these densities. The reader may refer to Vaughan $[5, \S \S 2.4$ and 2.6$]$ and Davenport $[3, \S 6]$ for the necessary techniques.

We now see that the conjectured number of solutions, without any adjustment for the failure of (WA), would be

$$
\mathfrak{S}_{k} N, \quad \mathfrak{S}_{k}=\sigma_{\infty, k} \prod \sigma_{p, k}
$$


To evaluate this more explicitly, we observe that, if $k$ is squarefree, then

$$
N\left(p^{n}\right)= \begin{cases}p^{3 n-3} N(p), & n \geq 1, p \nmid 3 k \\ p^{3 n-6} N\left(p^{2}\right), & n \geq 2, p \mid 3 k,\end{cases}
$$

by Hensel's Lemma. We can calculate $N(p)$ and $N\left(p^{2}\right)$ with the aid of cubic Gauss sums. We find that, for $p \nmid 3 k$, we have

$$
N(p)=p^{3}-1 \quad \text { if } p \equiv 2(\bmod 3),
$$

while for $p \equiv 1(\bmod 3)$ we have

$$
N(p)=p^{3}-1+3 p(p-1)\left\{(k / \pi)_{3}+(\overline{k / \pi})_{3}\right\}
$$

if $p$ splits as $\pi \bar{\pi}$, with $\pi \equiv 1(\bmod 3)$, in $\mathbb{Q}(\omega)$. When $p \mid k, p \neq 3$, we have

$$
N\left(p^{2}\right)=p^{6}-p^{4}
$$

and

$$
N\left(p^{2}\right)=p^{6}-p^{4}-p^{4}(p-1)(\pi+\bar{\pi})
$$

in the two cases. Finally,

$$
N\left(3^{2}\right)= \begin{cases}2 \cdot 3^{6}, & k \equiv \pm 1(\bmod 9), \\ 2^{2} \cdot 3^{5}, & k \equiv \pm 2(\bmod 9), \\ 2^{3} \cdot 3^{4}, & k \equiv \pm 3(\bmod 9), \\ 2 \cdot 3^{5}, & k \equiv \pm 4(\bmod 9) .\end{cases}
$$

The product defining $\mathfrak{S}_{k}$ is thus conditionally convergent only. However, by comparing it with the Euler product for $\zeta_{\mathbb{Q}(\sqrt[3]{k})}(s)$, we find that

$$
\mathfrak{S}_{k}=\sigma_{\infty, k} a_{k} b_{k}^{3} c \Pi_{1, k} \Pi_{2, k} \quad(k=2,3),
$$

where

$$
\begin{aligned}
& a_{2}=\frac{2^{9}}{3^{3} \cdot 7}, \quad a_{3}=\frac{2^{3}}{3^{2}} ; \quad b_{k}=\lim _{s \rightarrow 1}(s-1) \zeta_{\mathbb{Q}(\sqrt[3]{k})}(s) ; \\
& c=\prod_{p \equiv 2(\bmod 3)}\left(1-\frac{1}{p^{2}}\right)^{3}\left(1-\frac{1}{p^{3}}\right) ; \quad \Pi_{1, k}=\prod\left(1-\frac{1}{p}\right)^{7}\left(1+\frac{7}{p}+\frac{1}{p^{2}}\right),
\end{aligned}
$$

the last product being over primes $p \equiv 1(\bmod 3)$ which split in $\mathbb{Q}(\sqrt[3]{k})$; and

$$
\Pi_{2, k}=\prod\left(1-\frac{1}{p^{3}}\right)^{3}
$$

the product being for primes $p \equiv 1(\bmod 3)$ which do not split in $\mathbb{Q}(\sqrt[3]{k})$. The class number formula gives

$$
b_{k}= \begin{cases}\frac{\pi \log (1+\sqrt[3]{2}+\sqrt[3]{4})}{3 \sqrt{3}}, & k=2 \\ \frac{2 \pi \log (4+3 \sqrt[3]{3}+2 \sqrt[3]{9})}{9 \sqrt{3}}, & k=3\end{cases}
$$


Moreover, the products are now absolutely convergent and can be computed numerically to give

$$
\begin{aligned}
c & =0.30663 \ldots, \\
\Pi_{1, k} & = \begin{cases}0.98938 \ldots, & k=2, \\
0.98927 \ldots, & k=3,\end{cases} \\
\Pi_{2, k} & = \begin{cases}0.95401 \ldots, & k=2, \\
0.97617 \ldots, & k=3 .\end{cases}
\end{aligned}
$$

We may also calculate

$$
\sigma_{\infty, k}= \begin{cases}9.81424 \ldots, & k=2, \\ 8.57353 \ldots, & k=3,\end{cases}
$$

and

so that

$$
b_{k}= \begin{cases}0.814624 \ldots, & k=2 \\ 1.017615 \ldots, & k=3\end{cases}
$$

$$
\mathfrak{S}_{k}= \begin{cases}4.1598 \ldots, & k=2, \\ 2.3780 \ldots, & k=3 .\end{cases}
$$

For comparison one may find, by a naive computer search, all primitive solutions of $x^{3}+y^{3}+z^{3}=k w^{3}$ for $k=2,3$, in the region $|x|,|y|,|z| \leq 1000$. Of course, the solutions generally occur in multiples of 12 , because of the various symmetries involved. In searching for solutions one may use the restrictions found in Theorem 1, together with simple congruence conditions to moduli 2 , 7,9 , etc. In this way we find

$$
R_{2}(1000)=3906, \quad R_{3}(1000)=2462,
$$

so that

$$
\frac{R_{2}(1000)}{1000 \mathfrak{S}_{2}}=0.938 \ldots, \quad \frac{R_{3}(1000)}{1000 \mathfrak{S}_{3}}=1.035 \ldots .
$$

Figures 1 and 2 (see pages 618 and 619), which show $R_{k}(N)$ for $N \leq 1000$ together with the lines $\mathfrak{S}_{k} N$, indicate a linear growth rate in each case. The evidence therefore suggests that

$$
R_{k}(N) \sim \mathfrak{S}_{k} N
$$

despite the failure of (WA).

Although it seems hopeless to prove an asymptotic formula for $R_{k}(N)$, we can make a plausible heuristic investigation for suitably sized $K$ into the mean value $\sum_{K<k \leq 2 K} R_{k}(N)$. Allowing for solutions with $w=0$, we can write

$$
\sum_{K<k \leq 2 K} R_{k}(N)=6 K+2 \sum_{w=1}^{\infty} \# S(w),
$$

where

$$
\begin{aligned}
S(w)=\left\{(x, y, z) \in \mathbb{Z}^{3}:\right. & \operatorname{hcf}(x, y, z, w)=1,|x|,|y|,|z| \leq N, \\
& \left.w^{3} \mid x^{3}+y^{3}+z^{3}, K<\frac{x^{3}+y^{3}+z^{3}}{w^{3}} \leq 2 K\right\} .
\end{aligned}
$$




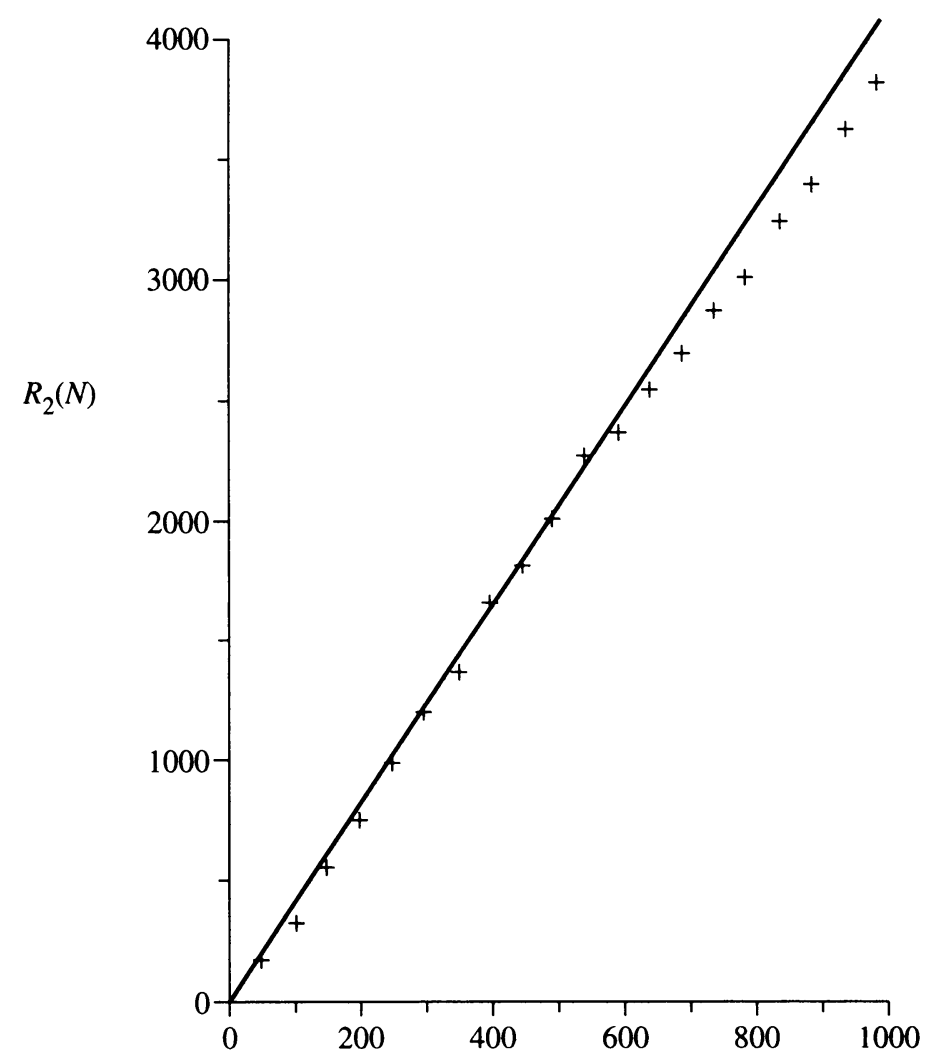

$N$

FIGURE 1

Of course, $S(w)$ is empty unless $K w^{3} \leq 3 N^{3}$. To estimate $\# S(w)$ in general, we observe that each admissible triple of residue classes for $x, y$, and $z$ modulo $w^{3}$ contains $V(w)+O\left(N^{2} w^{-6}\right)+O(1)$ elements, where

$$
\begin{aligned}
V(w)=\operatorname{meas}\left\{(x, y, z) \in \mathbb{R}^{3}:\right. & |x|,|y|,|z| \leq N w^{-3}, \\
& \left.K w^{-6}<x^{3}+y^{3}+z^{3} \leq 2 K w^{-6}\right\} .
\end{aligned}
$$

Let

$$
n(q)=\#\left\{x, y, z \quad(\bmod q): \operatorname{hcf}(x, y, z, q)=1, q \mid x^{3}+y^{3}+z^{3}\right\},
$$

so that $n\left(w^{3}\right)$ is the number of available triples of residue classes. Then

$$
n\left(w^{3}\right)=\prod_{p^{e} \| w^{3}} n\left(p^{e}\right)
$$

and

$$
n\left(p^{e}\right)=p^{2 e} \frac{n\left(p^{2}\right)}{p^{4}} \quad \text { for } e \geq 2,
$$

by Hensel's Lemma. We now define

$$
\rho(w)=\prod_{p \mid w} \rho(p), \quad \text { where } \rho(p)=p^{-4} n\left(p^{2}\right) .
$$




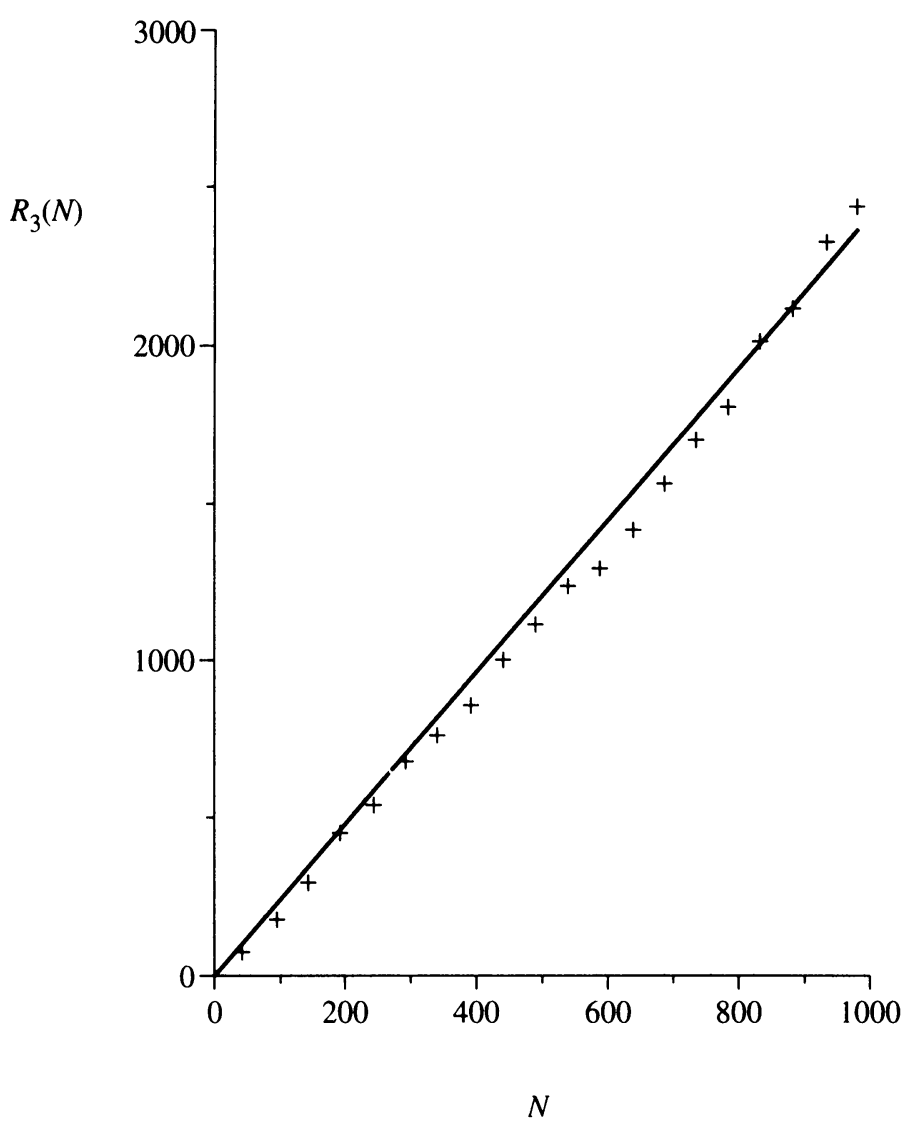

FIGURE 2

The Möbius inversion formula then yields

$$
\rho(w)=\sum_{d \mid w} \nu(d), \quad \text { where } \nu(d)=\mu(d) \prod_{p \mid d}(1-\rho(p)) .
$$

Since $n\left(p^{2}\right)=p^{4}+O\left(p^{7 / 2}\right)$, we have $\nu(d) \ll d^{-1 / 2+\varepsilon}$ for any $\varepsilon>0$, whence

$$
\begin{aligned}
\sum_{w \leq W} \rho(w) & =\sum_{d=1}^{\infty} \nu(d)\left[\frac{W}{d}\right]=W \sum_{d=1}^{\infty} \frac{\nu(d)}{d}+O\left(W^{1 / 2+\varepsilon}\right) \\
& =W \prod_{p}\left(1+\frac{\rho(p)-1}{p}\right)+O\left(W^{1 / 2+\varepsilon}\right) .
\end{aligned}
$$

We now have to examine

$$
2 \sum_{w} w^{6} \rho(w)\left\{V(w)+O\left(N^{2} w^{-6}\right)+O(1)\right\}
$$

the sum being over values $w \ll N K^{-1 / 3}$. According to (4), the error terms are $O\left(N^{3} K^{-1 / 3}\right)+O\left(N^{7} K^{-7 / 3}\right)$, whence (3) yields

$$
\sum_{K<k \leq 2 K} R_{k}(N)=2 \sum_{w} w^{6} \rho(w) V(w)+O(K)+O\left(N^{3} K^{-1 / 3}\right)+O\left(N^{7} K^{-7 / 3}\right) .
$$


We may write $V(w)$ as the difference $V_{2}(w)-V_{1}(w)$ of two monotonically decreasing functions given by

$$
\begin{aligned}
& V_{j}(w)=\operatorname{meas}\left\{(x, y, z) \in \mathbb{R}^{3}:|x|,|y|,|z| \leq N w^{-3}\right. \\
&\left.0 \leq x^{3}+y^{3}+z^{3} \leq j K w^{-6}\right\} .
\end{aligned}
$$

A partial summation then produces

$$
\begin{aligned}
\sum_{w} w^{6} \rho(w) V(w)= & \prod_{p}\left(1+\frac{\rho(p)-1}{p}\right) \int w^{6} V(w) d w \\
& +O\left(\max _{w \ll N K^{-1 / 3}} w^{1 / 2+\varepsilon} \cdot w^{6} \cdot V_{2}(w)\right) \\
= & \prod_{p}\left(1+\frac{\rho(p)-1}{p}\right) \int w^{6} V(w) d w+O\left(N^{1 / 2+2 \varepsilon} K^{5 / 6}\right) .
\end{aligned}
$$

The integral above is

$$
\begin{gathered}
\frac{1}{2} \int_{-\infty}^{\infty} w^{-3} \text { meas }\left\{(x, y, z) \in \mathbb{R}^{3}:|x|,|y|,|z| \leq N\right. \\
\left.K<\frac{x^{3}+y^{3}+z^{3}}{w^{3}} \leq 2 K\right\} d w \\
=\frac{1}{2} \int_{K}^{2 K} \int_{-N}^{N} \int_{-N}^{N} \int_{-N}^{N} \frac{d x d y d z}{\left(x^{3}+y^{3}+z^{3}\right)^{2 / 3}} \frac{d k}{3 k^{1 / 3}} .
\end{gathered}
$$

Thus,

$$
\sum_{K<k \leq 2 K} R_{k}(N) \sim \prod_{p}\left(1+\frac{\rho(p)-1}{p}\right) I\left\{\int_{K}^{2 K} \frac{d k}{k^{1 / 3}}\right\} N,
$$

providing that $N^{2+\delta} \leq K \leq N^{3-\delta}$ for some constant $\delta>0$. It seems likely that the lower bound for $K$ could be improved somewhat. We shall want to exclude values of $k$ which are cubes, $k=j^{3}$, say. However, if $x^{3}+y^{3}+z^{3}=j^{3} w^{3}$, then $z, j$, and $w$ determine $O\left(N^{\varepsilon}\right)$ values of $x$ and $y$, except when $z=j w$, in which case there are $O(N)$ pairs $x, y$. It follows that

$$
R_{j^{3}}(N) \ll j^{-1} N^{2+\varepsilon},
$$

whence such terms contribute $O\left(N^{2+2 \varepsilon}\right)$ to (5). It therefore follows that

$$
\sum_{K<k \leq 2 K}^{*} R_{k}(N) \sim \prod_{p}\left(1+\frac{\rho(p)-1}{p}\right) I\left\{\int_{K}^{2 K} \frac{d k}{k^{1 / 3}}\right\} N,
$$

for the same range of $K$ as before, where $\sum^{*}$ indicates that cube values of $k$ are omitted.

We shall now attempt to compare this with $N \sum_{K<k \leq 2 K}^{*} \mathfrak{S}_{k}$. When $k$ is not a cube, we write

$$
\mathfrak{S}_{k}=\sigma_{\infty, k} \lim _{X \rightarrow \infty} \prod_{p \leq X} \frac{N_{k}\left(p^{n(p, X)}\right)}{p^{3 n(p, X)}}=\sigma_{\infty, k} \lim _{X \rightarrow \infty} \frac{N_{k}(q(X))}{q(X)^{3}}
$$


where $n(p, X)=[\log X / \log p]$ and $q(X)=\prod_{p} p^{n(p, X)}$. If the convergence were uniform, we could write

$$
\sum_{K<k \leq 2 K}^{*} \mathfrak{S}_{k} \sim \sum_{K<k \leq 2 K}^{*} \sigma_{\infty, k} \frac{N_{k}(Q)}{Q^{3}},
$$

with $Q=q(\log \log N)$, say. Unfortunately, the convergence in (6) is not uniform. Nonetheless, the relation (7) seems plausible. Indeed, it seems likely that one could prove it with a little effort, and we therefore continue our investigation on the assumption that it does indeed hold.

We now observe that

$$
\begin{aligned}
\sum_{k \leq X}^{*} N_{k}(Q) & =\sum_{k \leq X} N_{k}(Q)+O\left(X^{1 / 3} N^{\varepsilon}\right) \\
& =\left(\frac{X}{Q}+O(1)\right) N^{*}(Q)+O\left(X^{1 / 3} N^{\varepsilon}\right)
\end{aligned}
$$

for any $\varepsilon>0$, where

$$
\begin{aligned}
N^{*}(s)=\#\{(x, y, z, w, k)(\bmod s): & \operatorname{hcf}(x, y, z, w, s)=1, \\
& \left.x^{3}+y^{3}+z^{3} \equiv k w^{3}(\bmod s)\right\} .
\end{aligned}
$$

Thus, summation by parts yields

$$
\sum_{K<k \leq 2 K}^{*} \sigma_{\infty, k} N_{k}(Q)=\frac{N^{*}(Q)}{Q} I \int_{K}^{2 K} \frac{d k}{k^{1 / 3}}+O\left(K^{-1 / 3} N^{*}(Q)\right)+O\left(N^{\varepsilon}\right) .
$$

Moreover,

$$
N^{*}(Q)=\prod_{p^{e} \| Q} N^{*}\left(p^{e}\right)
$$

and

$$
N^{*}\left(p^{e}\right)=N_{1}\left(p^{e}\right)+N_{2}\left(p^{e}\right),
$$

where $N_{1}\left(p^{e}\right)$ counts solutions with $p \nmid w$, and $N_{2}\left(p^{e}\right)$ counts those with $p \mid w$. Clearly,

$$
N_{1}=p^{4 e}\left(1-\frac{1}{p}\right),
$$

since $x, y, z, w$ determine $k$ uniquely. Moreover, Hensel's Lemma yields

$$
N_{2}\left(p^{e}\right)=p^{4 e} \frac{N_{2}\left(p^{2}\right)}{p^{8}},
$$

providing that $e \geq 2$ when $p=3$. Since, in our earlier notation, we have $N_{2}\left(p^{2}\right)=p^{3} n\left(p^{2}\right)=p^{7} \rho(p)$, we deduce that

$$
\frac{N^{*}(Q)}{Q}=Q^{3} \prod_{p \leq \log \log N}\left(1+\frac{\rho(p)-1}{p}\right),
$$


so long as $\log \log N \geq 9$. In view of (7), (8), and (9) we now have

$$
\begin{aligned}
\sum_{K<k \leq 2 K}^{*} \mathfrak{S}_{k} & \sim \frac{N^{*}(Q)}{Q^{4}} I \int_{K}^{2 K} \frac{d k}{k^{1 / 3}}+O\left(Q^{-3} K^{-1 / 3} N^{*}(Q)\right)+O\left(Q^{-3} N^{\varepsilon}\right) \\
& =\prod_{p \leq \log \log N}\left(1+\frac{\rho(p)-1}{p}\right) \int_{K}^{2 K} \frac{d k}{k^{1 / 3}}+O\left(N^{\varepsilon}\right) \\
& \sim \prod_{p}\left(1+\frac{\rho(p)-1}{p}\right) \int_{K}^{2 K} \frac{d k}{k^{1 / 3}}
\end{aligned}
$$

if $K \geq N^{\delta}$, for some constant $\delta>0$. We therefore have:

Theorem 2. If the conjecture (7) holds, then

$$
\sum_{K<k \leq 2 K}^{*} R_{k}(N) \sim N \sum_{K<k \leq 2 K}^{*} \mathfrak{S}_{k} .
$$

Thus, for the equation $x^{3}+y^{3}+z^{3}=k w^{3}$, all the evidence points to the conclusion that the Hardy-Littlewood formula is valid, despite the failure of (WA).

We conclude by investigating the Hardy-Littlewood formula for the equation $x^{3}+y^{3}+z^{3}=k$. The local densities will be

$$
\sigma_{p}=\lim _{n \rightarrow \infty} p^{-2 n} N\left(p^{n}\right)
$$

where

$$
N\left(p^{n}\right)=\#\left\{(x, y, z) \quad\left(\bmod p^{n}\right): x^{3}+y^{3}+z^{3} \equiv k \quad\left(\bmod p^{n}\right)\right\},
$$

while, for the range $N_{1} \leq \max (|x|,|y|,|z|) \leq N_{2}$, the real density of solutions is

$$
\begin{aligned}
\sigma_{\infty} & =6 \int_{N_{1}}^{N_{2}} \int_{0}^{z} \frac{d y}{3\left(z^{3}-y^{3}\right)^{2 / 3}} d z=2\left(\log \frac{N_{2}}{N_{1}}\right) \int_{0}^{1} \frac{d t}{\left(1-t^{3}\right)^{2 / 3}} \\
& =\frac{2}{3}\left(\log \frac{N_{2}}{N_{1}}\right) B\left(\frac{1}{3}, \frac{1}{3}\right)=3.5332 \ldots \log \frac{N_{2}}{N_{1}} .
\end{aligned}
$$

For cubefree $k$ we have

$$
\sigma_{p}=\frac{N(p)}{p^{2}} \quad(p \neq 3) \quad \text { and } \quad \sigma_{3}=\frac{N(9)}{81} .
$$

Then, as in our previous calculation, we obtain

$$
\sigma_{p}= \begin{cases}1, & p \nmid k, \\ 1-p^{-2}, & p \mid k,\end{cases}
$$

for $p \equiv 2(\bmod 3)$, and

$$
\sigma_{p}= \begin{cases}1+\frac{3}{p}\left\{\left(\frac{k}{\pi}\right)_{3}+\overline{\left(\frac{k}{\pi}\right)_{3}}\right\}-\frac{a}{p^{2}}, & p \nmid k, \\ 1+\frac{(p-1) a}{p^{2}}-\frac{1}{p^{2}}, & p \mid k,\end{cases}
$$


if $p \equiv 1(\bmod 3)$ splits as $\pi \bar{\pi}$ in $\mathbb{Q}(\sqrt{-3})$, and $4 p=a^{2}+3 b^{2}$ with $a \equiv 1$ $(\bmod 3)$. As before, we may compare $\sigma_{p}$ with the Euler factor $E(p, k)$ in $\zeta_{\mathbb{Q}(\sqrt[3]{k})}(s)$. We then find that

$$
\prod_{p} \sigma_{p}=A_{k}^{3} \prod_{p} \sigma_{p}^{\prime}
$$

where $A_{k}=\lim _{s \rightarrow 1}(s-1) \zeta_{\mathbb{Q}(\sqrt[3]{k})}(s)$, and $\sigma_{p}^{\prime}=\sigma_{p}((1-1 / p) / E(p, k))^{3}$. We now have a convergent product, and we can calculate

$$
\sigma_{\infty} \prod_{p} \sigma_{p}= \begin{cases}0.366 \ldots\left(\log N_{2} / N_{1}\right), & k=3, \\ 0.539 \ldots\left(\log N_{2} / N_{1}\right), & k=30,\end{cases}
$$

for example. In the light of our investigations above, we conjecture that these figures give, approximately, the number of solutions of $x^{3}+y^{3}+z^{3}=k$; and that no adjustment for the failure of (WA), as implied by Theorem 1 , is necessary. In particular, we conjecture that these Diophantine equations have infinitely many solutions. However, since the solutions have six symmetries, the density of essentially different solutions would be expected to be one-sixth of that indicated above. Thus, one might predict, very roughly speaking, one new solution of $x^{3}+y^{3}+z^{3}=3$ in the range

$$
N \leq \max \{|x|,|y|,|z|\} \leq \exp (6 / 0.366 \ldots) N \doteq 1.3 \times 10^{7} N
$$

and similarly, for $x^{3}+y^{3}+z^{3}=30$, one new solution in the range

$$
N \leq \max \{|x|,|y|,|z|\} \leq 7 \times 10^{4} N .
$$

A search for solutions to these equations (Gardiner, Lazarus, and Stein [4]) found only $(1,1,1)$ and $(4,4,-5)$, corresponding to $k=3$, and no solutions for $k=30$. Indeed, it was suggested that these equations have no more solutions. Inasmuch as the search range was only to $2^{16} \doteq 6.5 \times 10^{4}$, the figures above indicate that any such conjecture is premature. In particular, in the case of $k=3$, it would have been somewhat unexpected if a solution had been found.

\section{BIBLIOGRAPHY}

1. J. W. S. Cassels, $A$ note on the Diophantine equation $x^{3}+y^{3}+z^{3}=3$, Math. Comp. 44 (1985), 265-266.

2. J. W. S. Cassels and M. J. T. Guy, On the Hasse principle for cubic surfaces, Mathematika 13 (1966), 111-120.

3. H. Davenport, Cubic forms in thirty-two variables, Philos. Trans. Roy. Soc. London Ser. A 251 (1959), 193-232.

4. V. L. Gardiner, R. B. Lazarus, and P. R. Stein, Solutions of the Diophantine equation $x^{3}+y^{3}=z^{3}-d$, Math. Comp. 18 (1964), 408-413.

5. R. C. Vaughan, The Hardy-Littlewood circle method, Cambridge Univ. Press, 1981. 